\begin{tabular}{|c|l|}
\hline Title & $\begin{array}{l}\text { Reflection of cross disciplinary research at Creative Research Institution (Hokkaido University) in the Web of Science } \\
\text { database: appraisal and visual ization using bibliometry }\end{array}$ \\
\hline Author(s) & Gautam, Pitambar; Y anagiya, Ryuichi \\
\hline Citation & $\begin{array}{l}\text { Scientometrics, 93(1), 101-1111 } \\
\text { https://doi.org/10.1007/S11192-012-0655-3 }\end{array}$ \\
\hline Issue Date & 2012 \\
\hline Doc URL & http:/hdl.handle.net/2115/50158 \\
\hline Type & article (author version) \\
\hline File Information & Gautam and Yanagiya_SCIM648-2012-authorversion.pdf \\
\hline
\end{tabular}

Instructions for use 


\title{
Reflection of Cross-disciplinary Research at Creative Research Institution (Hokkaido University) in the Web of Science Database: Appraisal and Visualization using Bibliometry
}

\author{
Pitambar Gautam* and Ryuichi Yanagiya \\ Creative Research Institution, Hokkaido University, N21, W10, Kita-ku, Sapporo, \\ Hokkaido 001-0021, Japan
}

*Corresponding author

\begin{abstract}
This study describes the results of a preliminary bibliometric analysis of 611 research items, published between 1996 and 2011 by researchers affiliated with Creative Research Institution (CRIS) and the Center for Advanced Science and Technology (CAST), Hokkaido University (HU), retrieved from the Web of Science (WoS) database. CRIS has a primary mission to promote cutting-edge, world-class, trans-departmental research within $\mathrm{HU}$, and it conducts fundamental, commercialization-related, cross-disciplinary research and nurtures young in-house/recruited researchers through targeted, innovative tenure-track programs in multiple disciplines. Its research output derives from 3- to 7-year-long time-bound projects funded strategically by HU, external grants (e.g., MEXT Super-COE HU Research \& Business Park Project (FY2003-7)), industryuniversity collaboration with regional businesses, and endowments (e.g., Meiji Dairies). Analyses using co-words, bibliographic coupling, overlay map aided with visualization, etc., lead to the following inferences: (i) The published items comprise a dozen well-defined (inter-)disciplinary clusters, dominated by 3 macro-disciplines (biomedical science, $33 \%$; chemistry, $21 \%$; agricultural science, ca. 10\%) that constitute 18 clusters used for mapping; (ii) research conducted by externally funded or endowed projects in the biomedical, physical and environmental science \& technology fields ( 3 broad areas of aggregation derived from the Science Overlay Map) is interdisciplinary; and (iii) there is an apparently low visibility of publications from projects jointly executed with industries to an almost complete absence of output from CRIS in the fields of social sciences in the WoS database.
\end{abstract}

\section{Keywords}

Bibliometrics; Web of Science; Co-word analysis; Bibliographic coupling; Crossdisciplinary research; Science map; Japan

\section{Introduction}

Hokkaido University (HU) established Creative Research Initiative "Sousei" (CRIS) in 2002 as a University-wide organization to promote cutting-edge, worldclass research and to plan a mid- and long-term trans-departmental research system. After the incorporation of HU in 2004 and structural reforms, the Center for Advanced Science and Technology (CAST) was merged in FY2005 into CRIS, which then began to serve also as a base to materialize time-bound research schemes aimed at commercialization. In April 2009, CRIS was re-inaugurated as Creative Research Institution to serve also as a management organization with the 
mandate to coordinate 18 research-dedicated institutes, apart from giving continuity to the R\&D activities through its Research Department, which then comprised 5 horizontal divisions: frontier, innovative, strategic priority, projectbased (with businesses), and endowed. During FY2003-7, CRIS executed the MEXT Super-COE HU Research \& Business Park (HUR\&BP) Project. Fostering interdisciplinary exchanges, CRIS has a mission to promote research in an innovative and cross-disciplinary (in the broad sense, encompassing multidisciplinary, interdisciplinary, and transdisciplinary; we refer to Wagner et al. (2011) for a summary of key terms) manner aiming at knowledge creation and utilization.

In this paper, we present the first results of analyses on the research output of CRIS (publication volume, type, etc.) and outcome (e.g., citations and interdisciplinary contribution), partly to gauge the degree of reflection of the cross-disciplinary research output in the WoS database. Our aim was to assess the potential of bibliometry as an analytical tool for analysis and interpretation of the research performance of an institute having a complex mission at different levels of aggregation (e.g., institute, department, research groups).

\section{Publication data and methodology of analysis}

\section{Publication data}

Publications were harvested from the WoS SCI \& SSCI Database by searching for author affiliations including reprint address to CAST and CRIS. The search strings comprised phrases or abbreviations in addresses comprising basically 2 parts: (i) HOKKAIDO UNIV and (ii) CAST or C* ADV SCI TECH* or CRIS or SO\$SE\$ or C*EAT RES IN* or DIV INNOVAT RES or DIV FRONT RES. This was essential to take into account a dozen or more combinations representing a variety of styles, occasionally with misspelled variants for the Japanese name "SOUSEI" (meaning innovation or creation) and for the string "CREAT" for creative. The phrase CRIS BLDG had to be combined with logical NOT with (ii) in order to exclude references merely to the CRIS Building that also houses institutions other than CRIS. Because of the time-bound nature of the projects executed at CRIS and the very fact that CRIS used to be the secondary affiliation for many researchers, part of the research output resulting from research at CRIS might have been published with mere acknowledgement to CRIS, with another institute/department at $\mathrm{HU}$ as the only affiliation. Though the data retrieved may not be complete, we regard them to be fairly representative of the volume of research output from CAST and CRIS researchers.

Retrieval from WoS using the above-mentioned search strings on 15 January 2012 has yielded 643 citable items (articles, proceedings papers, reviews, and letters) jointly contributed by CAST and CRIS as illustrated in Fig. 1. The detailed analysis presented hereafter, however, is restricted to a total of 611 items retrieved on July 1, 2011 from the same database. There were 506 and 107 items with affiliation to CRIS (mainly from 2003 onward) and CAST (from 1996), respectively, implying that just 2 out of 611 items belonged to both affiliations. 


\section{Methods of analysis}

To assess the degree to which bibliometric analysis of publications recorded in the WoS database reflected the research activities at CRIS (including CAST), the following analyses were performed:

(i) Counting the frequency of assignment of items, and also of publications citing them, for different time spans to each of the 222 ISI science categories (SCs) and 18 macro-disciplines, following the procedure of Leydesdorff \& Rafols (2009). Both datasets were limited to records in WoS. Construction of a "Science Overly Map" using the SC counts following Rafols et al. (2010) aided by the Pajek 2.00 (1996-2010) program for visualization

(ii) Semantic analysis of co-words in titles of citable items separately for CAST and CRIS. Co-occurring words in titles of items were processed by using Bibexcel (Persson 2009) to generate a co-occurrence matrix comprising stemmed words. The matrix was subjected to a network analysis for clusters and mapping aided by visualization with VOSviewer (Van Eck \& Waltman 2010).

(iii) Analysis for bibliographic coupling of items in terms of journals in cited references to generate a cosine matrix by using BibJourn.exe (2011) by Loet Leydesdorff (pers. comm.), and further visualization as a 2D network map by using Pajek.

\section{Results of the bibliometric analysis}

\section{Degree of coverage of various scientific disciplines}

Figure 2 summarizes the citable items for 2002-2010, a time span representative for CRIS, in terms of the disciplinary coverage based on the assignment of each item to one of the macro-disciplines (equivalent to 18 clusters in the overlay base map) obtained by summing up frequencies of occurrence in several SCs by using the fractional counting method (for percentage data, see second column in Table 1). Three macro-disciplines represented by Biomedical Science (32.8\%), Chemistry (19.2\%), and Agriculture Sciences (12.0\%) covered more than half (54\%) of the items. Significant contributions varying from $8.7 \%$ to $4.1 \%$ from 5 other macro-disciplines (Ecological Science, Materials Science, Physics, Infectious Diseases, and Cognitive Science) comprised another 30\%. Further aggregation at a higher level to 4 broad fields revealed the share of Biomedical Sciences, Physical Sciences and Environmental Science \& Technology (see Fig. 2) to be $43.0 \%, 33.5 \%$, and $23.3 \%$, respectively, whereas the Social Sciences category or field was almost absent.

Calculation of the share of macro-disciplines for the 2002-2010 dataset discussed previously was repeated for additional time spans, including 2005-2008 as the most representative period for output of the HUR\&BP project and the most productive one for CRIS, after merging of CAST into CRIS (Table 1). The dataset was extended to include 3,106 items (also citable) citing the 2005-2008 output (329 items), to gain better insight into interdisciplinarity (Porter et al. 2007). Comparison of the datasets in 4 different columns in Table 1 reveals a remarkable similarity in the share of most macro-disciplines, with an exception of minor differences for Agricultural Science and Cognitive Science. 


\section{Appraisal of cross-disciplinary coverage through a science map}

Based on the overall similarity in the disciplinary share discussed above, we feel any dataset used to create Table 1 to be suitable for construction of a Science Overlay Map to visualize the internal dynamics of the research output (publications) or outcome (citations). To gain overall insight for both CAST and CRIS, all 611 items were assigned to individual SCs without fractionation (i.e., if an item was assigned to $3 \mathrm{SCs}$, each of them received a score of 1 rather than 1/3) to draw a 2-dimensional map (Fig. 3). In the map, macro-disciplines and SCs with a predominant share (percent relative to 611 items) were identified as follows:

a) Biomedical Science: Biochemistry \& Molecular Biology (20.1\%); Genetics \& Heredity (8.7\%); Biophysics (7.4\%); Biotechnology \& Applied Microbiology $(7.0 \%)$.

b) Materials Science: Polymer Science (9.3\%); Material Science, Multidisciplinary $(4.9 \%)$.

c) Chemistry: Chemistry, Physical (6.1\%); Chemistry, Multidisciplinary (5.2\%).

d) Agricultural Sciences: Plant Sciences (6.7\%), Food Science \& Technology $(4.7 \%)$.

The map is advantageous in its power to discriminate SCs with a minor contribution (e.g., Paleontology, with $<0.5 \%$ of the total output; top right in Fig. 3 ). Whole counting, however, variably enhanced the visibility of individual SCs and macro-disciplines in the map compared to the use of fractional counting used to generate data in Table 1. The score for 'Multidisciplinary Science' comprising specific set of journals assigned only to this SC remains unaffected by the counting method. For other SCs contributing at least 1\% of the 611 items using fractional counting, whole counting enhanced the share by a factor of 1.10 (for Zoology) to 3.37 (for Chemistry, Applied), with an average of 1.73. At the level of macro-discipline, Physics and Ecological Science exhibited the least enhancement (ca. 1.3), whereas a maximum value of ca. 2.1 characterized Chemistry and Clinical Medicine.

\section{Co-word analysis and visualization of clusters}

Datasets from CAST and CRIS were analyzed for co-words in titles separately to reveal distinctive peculiarities. Selected words and 2-D visualizations of their cooccurrence relations forming clusters are presented in Tables $2 \& 3$ and Fig. 4. Vosviewer allowed zooming and viewing specific areas as color density maps, enhancing readability and interpretation.

Research outcomes from activities initiated at CAST, and continued at CRIS for some time (mainly as former next-generation post-genome and genome dynamics research divisions, during FY2005-2008) were as follows: chromosome-based molecular cytogenetic/karyotypic studies of animals (clusters 1, 6 in dataset 1 and clusters 8,10 in dataset 2), phylogeny of mammals through ancient and modern DNA analyses (cluster 3 in dataset 1), and taxonomic/phylogenetic characterization of red and green algal species (cluster 4 in dataset 1). Cluster 7, protein-structure analysis, was another prominent theme in both datasets. 
The following most productive fields (corresponding clusters in dataset 2, Fig. 4b) were clearly evident for CRIS: synthesis, and characterization of polymers/macromolecules/gels (cluster 1); photochemical fabrication of nanodevices using molecular crystals or organic materials (cluster 2); human cell culture using fish collagen and production of honeycomb film scaffold for transplantation (cluster 3); rhizosphere - the plant root \& soil environment and new cultivation system (cluster 4); zoonotic diseases including avian influenza (cluster 5); gastrointestinal tract/biosphere and functional enzymes/foods, etc. (cluster 6); and, genomic expression in sea squirts (clusters 9\& 10) from the world's oceans.

\section{Coupling pattern of journals as indicators of scientific specialties}

The complete dataset $(\mathrm{N}=611)$ was analyzed for bibliographic coupling in terms of journals in the cited references that serve as the knowledge base; this also provided information on scientific disciplines. Of the 20,834 total references cited, 11,253 belonged to 2,696 journals indexed in WoS. Visualization in a 2D map involved running bibJourn.exe at a threshold of $0.2 \%$ (only journals appearing in at least 22 cited references) to obtain a matrix of Salton cosines, and further processing with Pajek after partition.

Figure 5 shows the mutual arrangement of 93 coupled journals (in cited references). Ten journals (each provided with corresponding frequency) that topped the list of the knowledge source consulted by (co-)researchers from CAST and CRIS were: Nature (255), Science (245), P Natl Acad Sci USA (204), J Biol Chem (147), J Am Chem Soc (96), Cell (82), Nucleic Acids Res (80), Phys Rev Lett (77), J Mol Biol (75), and Biochem Biophys Res Co (74). The first 3 journals belong to the "Multidisciplinary Sciences" according to the ESI 22-field division, but are included in the "Biomedical Science" macro-discipline (Fig. 3) in the 18cluster classification we have used (Fig. 3). The size of each node labeled on the map reflects the frequency of citations of journals. The mutual separation of nodes increases with decreasing coupling relationship, and the thickness of connecting lines indicates the strength of the joint relationship. In Fig. 5, three important groups of journals are enclosed by ovals. The area designated roughly as 'biomedical and agricultural sciences' is very broad and surely exhibits an interdisciplinary nature.

\section{Discussion and conclusion}

The percentage share of the 2002-2010 research outcome from CRIS (partially also from CAST) in terms of 222-ISI SCs, 18-macro-disciplines, and further aggregation into 4 fields (Biomedical Sciences, Physical Sciences, Environmental Science \& Technology, and Social Sciences) using the fractional counting method revealed that 3 fields in Science \& Engineering comprise almost the whole dataset, with a fairly large share for each (varying between 23 and 45\%); the Social Sciences field was virtually absent (Table 1). An overlay science map revealed the disciplinary structure at the level of SCs, again showing the clear dominance of Biomedical Science (including 6 items or only a 1\% share of the Multidisciplinary Sciences category), Material Science, Chemistry, and Agricultural Sciences. 
Co-word analyses for titles separately for CAST and CRIS revealed clusters such that the word assemblies allowed recognition of research themes leading to the production of an appreciable number of publications in journals recognized and indexed in the WoS database.

Themes marked by clusters simultaneously in the CAST and CRIS data (Tables $2 \& 3$; Fig. 4a, b) on genetic/genomic and protein structure analyses were indeed interdisciplinary, as evidenced by significant overlaps among the clusters. The genetic/genomic studies involved close collaboration among researchers from the Graduate Schools of Science, Advanced Life Science, and Agriculture as well as many researchers from institutes abroad, and the objects studied (e.g., animals, algal species) have global coverage (Hokkaido University 2011). The proteinstructure studies focused on trailblazing research on DNA-protein-sugar chain mechanisms, and the findings served as the basis for collaboration between HU and businesses (e.g., companies engaged in the medical care/pharmaceutical sector) for drug development/discovery R\&D projects. Most of these studies are currently being continued at the Genome Dynamics Research Center (since 2008) and the Frontier Research Center for Post-Genome Science \& Technology (mainly since 2007) affiliated with the Faculty of Science and the Faculty of Advanced Life Science, respectively (Hokkaido University 2011).

Clusters identified from the CRIS data (Table 3; Fig. 4b) reflect very well the most productive projects (clusters $2,4,9,10$ ), led by 3 young researchers nurtured at CRIS's Innovative Research Division and one of the two frontier research programs. Likewise, results of the strategically promoted interdisciplinary teambased research conducted under the HUR\&BP Project on (i) Development of Diagnosis \& Treatment of Zoonosis, (ii) Tissue Transplantation \& Engineering, and (iii) Safe \& Sustainable Food Production define clusters 5, 3, and 6, respectively (Hokkaido University2011). Team research on tissue regeneration and bio/nanotechnology-based medical care pursued in the Frontier Research Division also contributed significantly to the study of polymers/biomaterials (Materials Science).

A bibliographic coupling analysis (Fig. 5) showed that almost all journals serving as the most representative knowledge base for our dataset belong to the Biomedical and Agricultural (including some related to environment) Sciences, Chemistry, and Materials Science, reinforcing the similarity with findings from disciplinary assignments and the co-word analyses. Other notable SCs (macrodisciplines) detected from a joint analysis of all previous data, although comparatively less productive, seem to be Physics (see Figs. 3, 5), Neurosciences (Cognitive Science), and Geochemistry \& Geophysics (Geosciences). These disciplines have been actively pursued by the frontier research team dealing with 'molecular/structural simulations of superconductors', the $4^{\text {th }}$ innovative research project at CRIS, and the Isotope Imaging Laboratory in the Strategic Project Research Division, respectively. These themes are partly representative of the trans-disciplinary research carried out as innovative projects by young researchers and by other programs that engaged researchers transcending various departments and also businesses. 
Comparison of data for different time spans at the level of each macro-discipline (Table 1) reveals relatively lower values for Agricultural Science and Cognitive Science in the last column for items citing 329 publications for 2005-2008. Such low values may have resulted from the comparatively low annual volume of publications in these 2 macro-disciplines during the period concerned.

Publications in Agricultural Science showed an already clearly increasing trend from 2005, whereas Cognitive Science showed a slight recovery after an almost lack of publications during 2007-2008. As these trends in the publication volume were dictated by several factors (e.g., completion of the HUR\&BP Project in April 2008; continued funding to a few selected projects), these minor variations are not important. However, a longer period (e.g., at least 5-6 years) could be more representative if the aim were to judge the average productivity and impact at the level of an institute.

An almost complete absence of items in Social Sciences in the WoS records was clear, even though CRIS had a few research programs engaged partly in this field, i.e., the Environmental Science \& Technology Policy Program under HUR\&BP and 2 research projects in the Innovative Research Division (Hokkaido University, 2011). Filling the information void in social sciences would require consulting non-WoS journals (including those written in Japanese) and other modes of publications (e.g., monographs). It remains to be seen to what extent such an approach would be necessary for a fuller appreciation of the research output in other science and engineering fields.

The analyses and interpretations attempted so far, though largely descriptive, have demonstrated the usefulness of bibliometry as a tool for recognizing the comprehensive knowledge structure (output and outcome) at the level of an institute at Hokkaido University. Further analyses using other network tools that combine several different pieces of information (e.g., word-author-journal) and $2 \mathrm{D}$ visualization as time-series maps will surely give insight into shifting tendencies in research practice, emerging disciplines, etc., and thus provide data useful for strategic planning.

CRIS is an institute with the mission of promoting/conducting cross-disciplinary research, and the body of knowledge already produced there indeed involved teamwork and collaboration. However, it is desirable to apply indicator-based methods, such as those suggested by Leydesdorff \& Rafols (2011) and Wagner et al. (2011), to judge the extent of multidisciplinarity (simple integration), interdisciplinarity (a holistic approach with input from various disciplines leading to partial/complete fusions, novel syntheses, etc.), and transdisciplinarity (extending beyond or crossing the restricted boundaries of constituent disciplines, research participants, etc.) in quantitative/statistical terms.

Finally, we emphasize that there is a clear need for applying bibliometric data objectively, with due consideration of the mission, structure, temporal characteristics, etc., of the research establishment in question.

\section{Acknowledgments}

We are grateful to I. Uyeda and H. Okada, the current and former Executive Directors of CRIS, whose advices and opinions during discussions provided insights for interpretation. We thank 
Matthew H Dick, Professor at the Graduate School of Science, for many comments to achieve clarity in interpretation and the linguistic improvements. We were encouraged very much by $\mathrm{T}$. Ogawa, Deputy Director of CRIS, to perform this experimental analysis on the CAST and CRIS datasets. It is to emphasize, however, that we as authors are solely responsible for the approach, analyses, and interpretations presented in this paper.

\section{References}

BibJourn.exe (2011). A program for cocitation analysis of journal names in references written by Loet Leydesdorff. Downloadable from: http://www.leydesdorff.net/software/bibjourn/index.htm

Hokkaido University (2011). Hokkaidou daigaku souseikennkyuukikou 9-nenkan no ayumi (Nine years of Creative Research Institution (CRIS), 125+ pp. (Internal publication of Hokkaido University, Sapporo, Japan, in Japanese)

Leydesdorff, L. \& Rafols, I. (2009). A global map of science based on the ISI subject categories. Journal of the American Society for Information Science and Technology, 60(2), 348-362.

Leydesdorff, L. \& Rafols, I. (2011). Indicators of the interdisciplinarity of journals: Diversity, centrality, and citations. Journal of Informatics, 5(1), 87-100.

Persson, O. (2009). Bibexcel - A toolbox for bibliometricians. Version 2009-11-26. Downloaded from: http://www8.umu.se/inforsk/Bibexcel/index.html

Pajek 2.00 (1996-2010) developed by Vladimir Batagelj and Andrej Mrvar. Freely available for non-commercial use from: http://pajek.imfm.si/

Porter, A.L., Cohen, A.S., Roessner, J.D. \& Perreault, M. (2007). Measuring researcher interdisciplinarity. Scientometrics, 72(1), 117-147.

Rafols, I., Porter, A.L. \& Leydesdorff, L. (2010). Science overlay maps: A new tool for research policy and library management. Journal of the American Society for Information Science and Technology, 61(9), 1871-1887.

Van Eck, N.J. \& Waltman, L. (2010). Software survey: VOSviewer, a computer program for bibliometric mapping. Scientometrics, 84, 523-538. Program downloadable from:

http://www.vosviewer.com/

Wagner, C.S., Roessner, J. D., Bobb, K,, Klein, J.T., Boyack, K.W., Keyton, J., Rafols, I. \& Börner, K. (2011). Approaches to understanding and measuring interdisciplinary scientific research (IDR): A review of the literature. Journal of Informetrics, 165, 14-26.

\section{Figures with Captions}




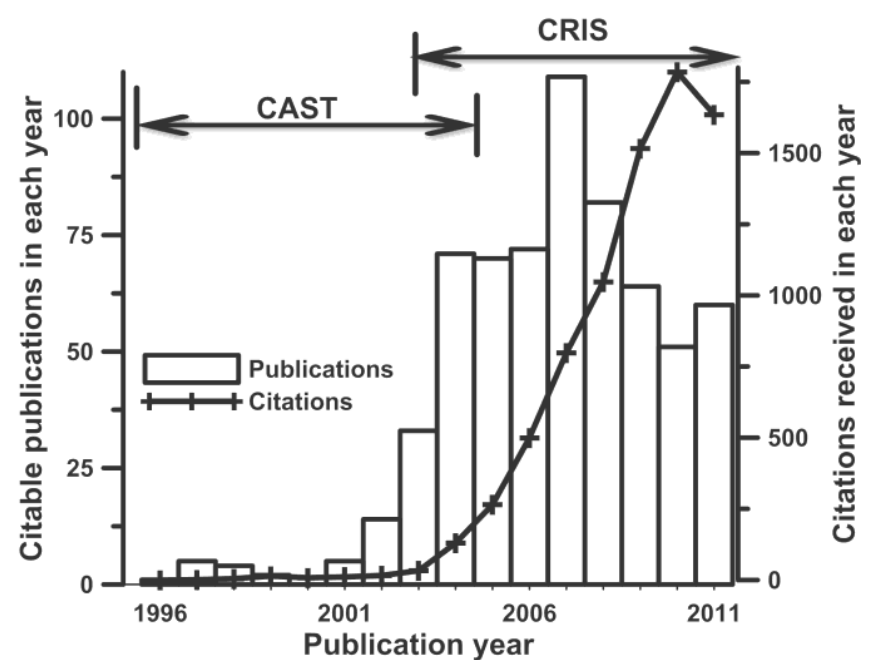

Fig.1 Annual distribution of 643 citable publications with (co-)author affiliations to CAST \& CRIS, HU, and their citations each year retrieved from WoS on January 15, 2012. Period applicable: 1996 to 2011

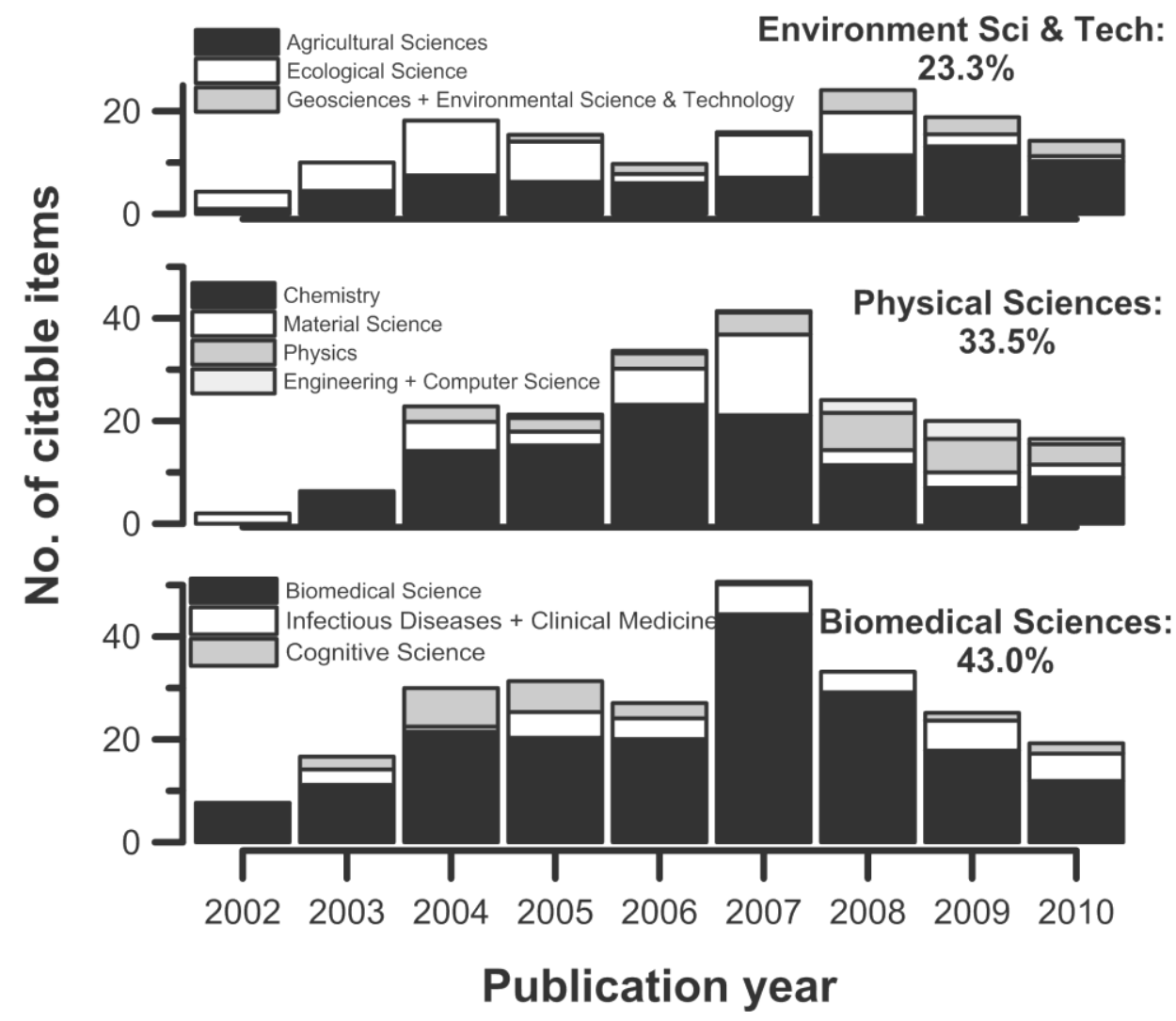

Fig. 2 Breakdown of the 2002-2010 citable items into macrodisciplines (parts of 18 clusters) and 4 broad field categories (Leydesdorff \& Rafols, 2009). The Social Sciences category (not shown here) has a share of merely $0.2 \%$. Number of items analyzed: 561 
Table 1 Breakdown of the citable items from CAST and CRIS, and the publications citing the items, into macro-disciplines following Leydesdorff \& Rafols (2009)

\begin{tabular}{|c|c|c|c|c|}
\hline \multirow{3}{*}{$\begin{array}{c}\text { Macro-discipline } \\
\text { (cluster based on } \\
\text { co-citations of ISI SCs) }\end{array}$} & \multicolumn{4}{|c|}{$\%$ share of each macro-discipline in } \\
\hline & \multicolumn{3}{|c|}{$\begin{array}{c}\text { Items published } \\
\text { (Articles, Reviews, Proc papers, Letters) }\end{array}$} & \multirow{2}{*}{ 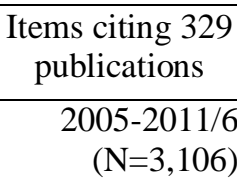 } \\
\hline & $\begin{array}{r}1996-2011 / 6 \\
(\mathrm{~N}=611)\end{array}$ & $\begin{array}{r}2002-2010 \\
(\mathrm{~N}=561)\end{array}$ & $\begin{array}{l}2005-08 \\
(\mathrm{~N}=329)\end{array}$ & \\
\hline Biomedical Science & 33.0 & 32.8 & 34.6 & 32.0 \\
\hline Chemistry & 19.2 & 19.2 & 21.6 & 23.9 \\
\hline Agricultural Science & 11.0 & 12.0 & 9.3 & 6.4 \\
\hline Ecological Science & 8.6 & 8.7 & 8.0 & 8.5 \\
\hline Materials Science & 7.3 & 7.4 & 8.6 & 8.0 \\
\hline Physics & 6.6 & 5.5 & 5.2 & 5.4 \\
\hline Infectious Diseases & 4.4 & 4.2 & 4.1 & 5.3 \\
\hline Cognitive Science & 3.8 & 4.1 & 2.9 & 1.9 \\
\hline Clinical Medicine & 2.1 & 1.9 & 1.6 & 3.7 \\
\hline Geosciences & 1.8 & 1.9 & 1.7 & 2.5 \\
\hline Engineering & 1.0 & 1.3 & 1.1 & 0.7 \\
\hline $\begin{array}{l}\text { Environmental Science } \\
\text { and Technology }\end{array}$ & 0.6 & 0.7 & 0.8 & 0.4 \\
\hline Computer Science & 0.2 & 0.2 & 0.1 & 0.6 \\
\hline Others ( 5 categories) & 0.2 & 0.2 & 0.4 & 0.6 \\
\hline
\end{tabular}

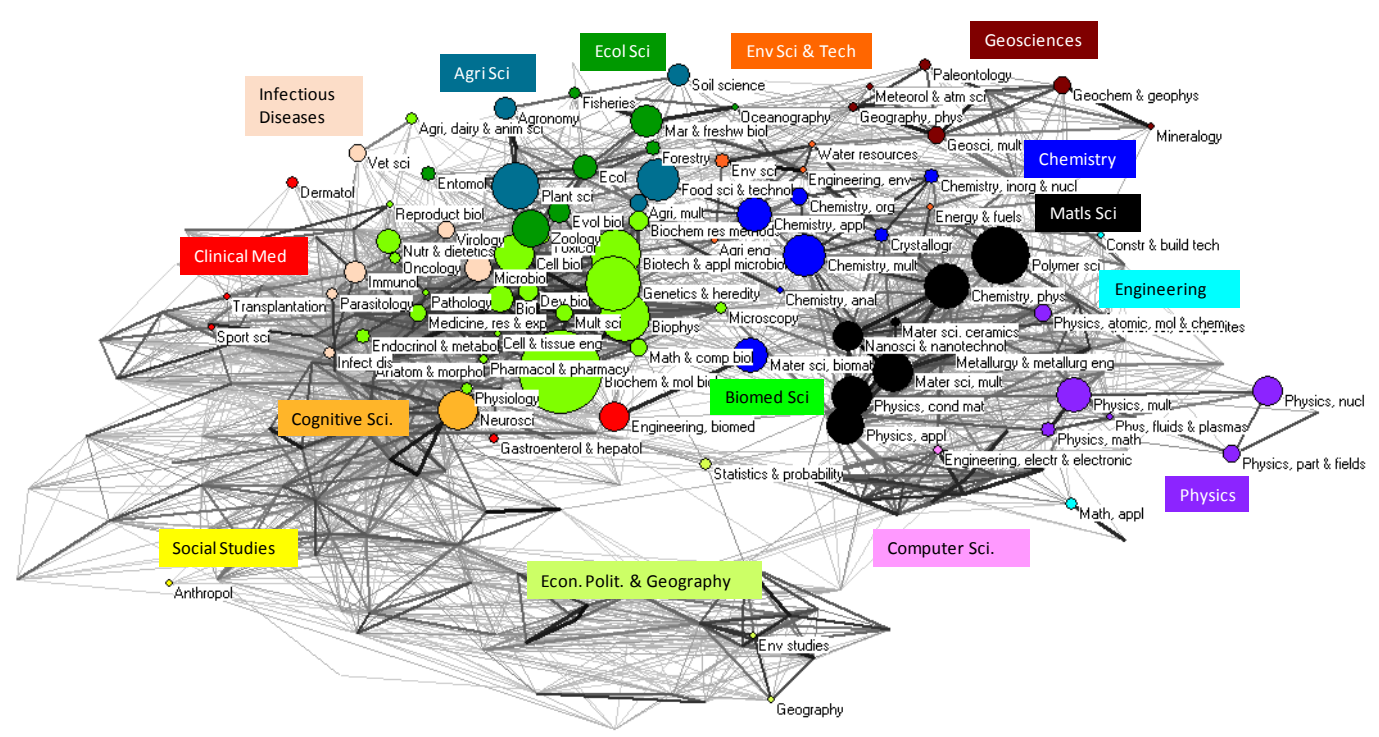

Fig.3 Science Overlay Map derived from 611 citable items from CAST \& CRIS. The base map with labelled nodes (some of the 222 ISI SCs) is from Rafols, Porter \& Leydesdorff (2010). The nodal diameter reflects item counts. The separation of the nodes and the connecting lines were taken from the Global Science Map 2009. Macro-disciplines are shown by the shaded boxes 
Published online: 11 February 2012 in Scientometrics, DOI 10.1007/s11192-012-0655-3

Table 2 Seven clusters, each being defined by 5 co-occurring terms (selected from the set of 10 most frequent stemmed words) that reflect the most productive research themes from CAST

Dataset 1: 107 items (CAST)

Cluster No. \& stemmed words: Fig. 4(a)

1: chromosom, gene, mous, chicken, fish

2: sequenc, genet, mitochondri, divers, speci

3: popul, hokkaido, brown, bear, deer

4: japan, red, solieriacea, rhodophyta, gigartinal

5: rat, salmon, structur, cell, transgen

6: molecular, karyotyp, cytogenet, evolut, shrew

7: dna, pharaoni, bind, halorhodopsin, protein

Table 3 Eleven clusters, each represented by 5 co-occurring terms (selected from the set of 10 most frequent stemmed words) that reflect the most productive research themes from CRIS

Dataset 2: 506 items (CRIS)

Cluster No. \& stemmed words: Fig. 4(b)

1: synthesi, hydrogel, polym, surfac, network

2: molecular, structur, crystal, organ, cluster

3: cell, human, film, collagen, gel

4: rice, phosphoru, growth, metabol, tropic

5: mous, influenza, viru, h5n1, pathogen

6: gene, function, sequenc, isol, bacteri

7: protein, differenti, bind, neuron, signal

8: chromosom, sex, dna, induc, bird

9: carbon, microarrai, hybrid, ascidian, marker

10: intestinali, ciona, transcript, cycl, specif,

11: dynam, calcium, hydrat, first, ion 

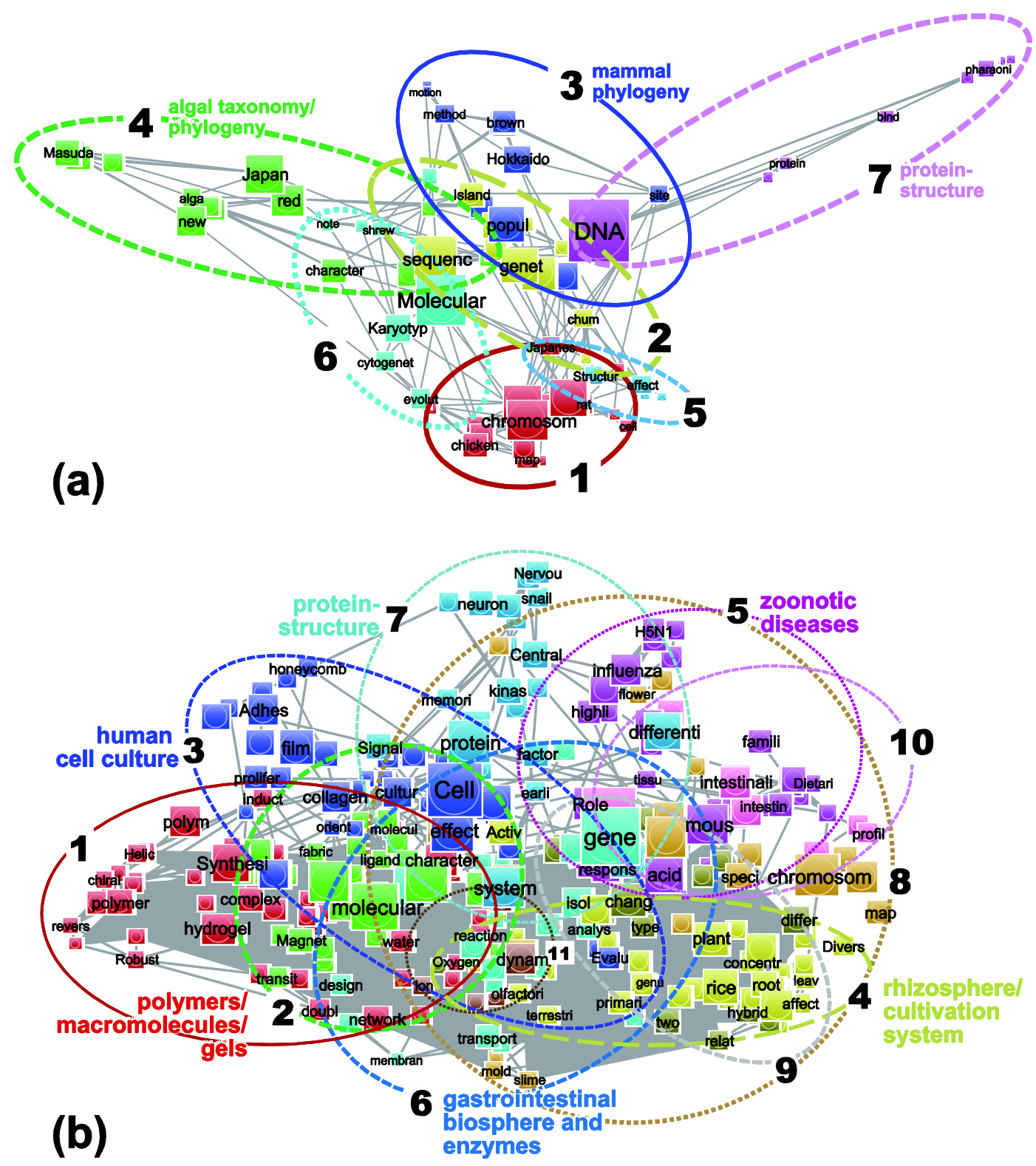

Fig.4 (a, b). Density label maps to visualize bibliometric mapping and the clustering of research output based on co-occurring words in titles of the citable items. Stemmed co-words were processed by Bibexcel (Persson 2009) to generate co-occurrence matrices that were clustered and mapped with VOSviewer. The size of each node (solid circle) and the size and contrast of the corresponding label are proportional to the assigned weight. Selected clusters are given theme labels. (a) 107 items from CAST (7 clusters, with slight overlap). Minimum word frequency $=3$. (b) 506 items from CRIS (11 clusters, with significant overlap across several of them). Minimum word frequency $=4$. For terms defining each cluster, refer to data in Tables 2 and 3. 


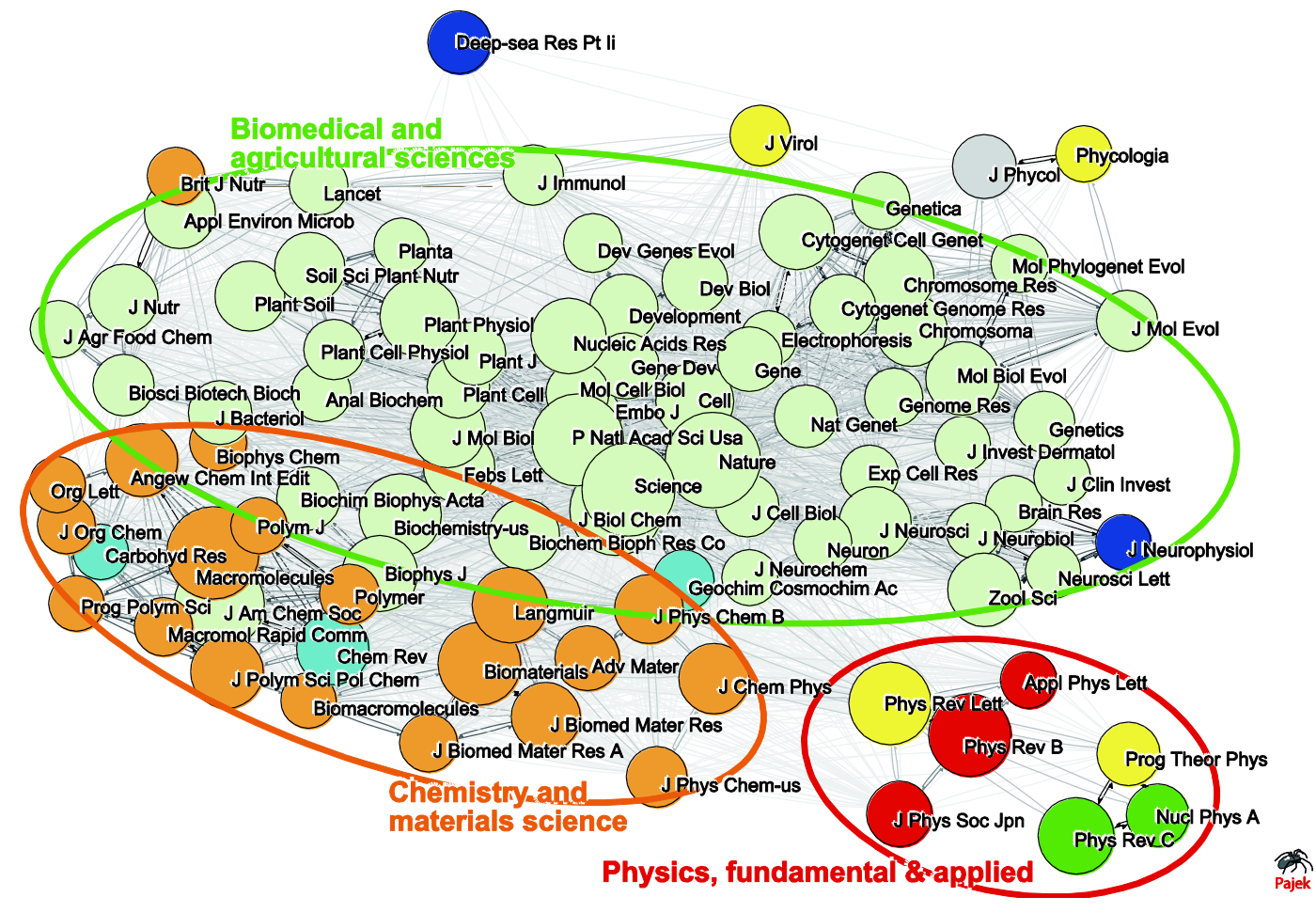

Fig. 5 2D visualization of bibliographic coupling of the 93 most frequent journals, as the knowledge base, in cited references of 611 items. Ovals delineate 3 generalized groups, definition of which was not restricted any particular categorization 